\title{
Atención de Pacientes en Fase Terminal. Experiencia de la Unidad de Cuidados Paliativos del Hospital Max Peralta de Cartago durante 1996
}

\author{
Juan C. Irola Moya
}

\begin{abstract}
Resumen
La Unidad de Cuidados Paliativos de Cartago pertenece al Hospital Max Peralta; es un equipo interdisciplinario que brinda atención a personas con enfermedades terminales y sus familias, basados en los principios de los cuidados paliativos. Inició su trabajo en abril de 1990, siendo el primer grupo en el país en asumir la atención de pacientes con enfermedad terminal y de sus familias.

Este artículo describe las actividades mas relevantes realizadas por la Unidad durante 1996.

De un total de 154 pacientes, durante el año fallecieron 82, encontrándose que el mayor número de usuarios de la Unidad fueron personas mayores de 61 años, con un ligero predominio de hombres. Los diagnósticos de ingreso más frecuentes fueron los tumores malignos del aparato digestivo, siendo los pacientes referidos por diferentes servicios del Hospital Max Peralta y del Hospital Calderón Guardia

Se registraron los síntomas de los pacientes al ingresar a la Unidad, encontrándose que el dolor fue el principal.

La atención que se brindó fue en el nivel domiciliar, teniendo cada paciente un promedio de tres visitas domiciliares y por lo menos tres sesiones familiares.

Se logró evidenciar el impacto de la intervención de la Unidad en dos aspectos:

1- El éxito alcanzado en el alivio del dolor: el 52.4. \% de los pacientes fallecieron sin dolor y el $31.7 \%$ con dolor leve

2- La necesidad de hospitalización de los pacientes atendidos que fue mínima: 5 pacientes (6.1\%). (AMC, 1998; 40 (1): 20-
\end{abstract} 26)

\section{Introducción}

Los cuidados paliativos han tomado fuerza en los últimos años en nuestro país con el surgimiento de varios grupos de carácter interdisciplinario dentro de algunos hospitales de la Caja Costarricense de Seguro Social.

Los cuidados paliativos plantean como fin fundamental la atención de personas que enfrentan una enfermedad terminal, considerándolas en el marco de su interrelación familiar y comunitaria, esto con el propósito de construir conjuntamente,

*Médico Asistente especialista en Medicina Familiar y Comunitaria, Area de Salud del Guarco.

marzo 1998, AMC, vol. 40 (1) paciente-familia-comunidad y equipo de cuidados paliativos, un proceso centrado en el concepto de calidad de vida, para que la muerte como desenlace final sea lo menos traumática y dolorosa posible ${ }^{1,2}$.

Para lograr lo anterior se parte de un enfoque de atención biopsico-social-espiritual, que implica necesariamente un trabajo en equipo en el que participan diferentes disciplinas, tales como: medicina, enfermería, trabajo social, psicología, farmacia, guías espirituales y voluntariado.

Por otro lado, siendo el control de síntomas y el control del dolor una de las actividades más importantes que se realizan en cuidados paliativos, la Organización Mundial de la Salud, (OMS), en el informe técnico $804^{3}$, establece un esquema escalonado para el control de dolor por cáncer, que marcó una pauta importante en el abordaje, manejo y tratamiento farmacológico del paciente.

En el esquema mencionado la $\mathrm{OMS}^{3}$, establece tres categorías de dolor: leve, moderado y severo, recomendando el uso de analgésicos no opioides con coadyuvantes o coanalgésicos, en el primer paso; en el segundo paso opiodes débiles más analgésicos no opiodes más coadyuvantes, y opiodes fuertes más analgésicos no opioides con coadyuvantes para el dolor severo, como tercer paso.

Para señalar a una persona como paciente terminal se debe considerar la presencia de los siguientes aspectos ${ }^{1.2}$ :

1. Presencia de una enfermedad avanzada, progresiva, incurable.

2. Falta de posibilidades razonables de respuesta al tratamiento específico.

3. Presencia de numerosos problemas o síntomas intensos, múltiples, multifactoriales y cambiantes.

4. Gran impacto emocional en el paciente, la familia y el equipo terapéutico, muy relacionado con la presencia, explícita o no, de la muerte.

5. Pronóstico de vida inferior a seis meses.

\section{Materiales y Métodos}

Para obtener los datos que se muestran en este artículo se aplicó, a su ingreso, un cuestionario a todos los pacientes atendidos por la Unidad de Cuidados Paliativos durante el año de 1996, cuyo total fue de 154 pacientes (universo); para 
efectos del análisis se tomó en cuenta unicamente a los pacientes fallecidos durante el año, los que sumaron 82 (muestra).

El cuestionario fue diseñado y aplicado por los miembros de la Unidad, siendo el mismo previamente codificado y probado. La información se obtuvo de tres fuentes: entrevista a 9 pacientes, entrevista a familiares y revisión de expedientes clínicos.

El cuestionario incluyó las siguientes variables sociodemográficas, lugar de referencia, diagnóstico de ingreso, registro de síntomas, registro de medicamentos analgésicos y coadyuvantes utilizados, vías de aplicación y tipo de intervención como visita domiciliar y clínicas de familia. Además, se registró si el paciente ameritó o no hospitalización durante el tiempo que permaneció al cuidado de la Unidad. En el registro de síntomas se hizo énfasis en el dolor, registrándose al ingreso y durante todo el tiempo que el paciente fue asistido por la Unidad; para ello utilizamos la escala visual numérica, diseñandose una hoja especial para que el paciente o su familia registraran la intensidad del dolor, diariamente y por lo menos una vez, previo entrenamiento. Utilizamos una escala numerada de 0 a 10 , cuya interpretación fue: 0 sin dolor, $1-3$ dolor leve, 4-6 dolor moderado, 7-10 dolor severo.

Para efectos de la investigación consideramos unicamente el registro del dolor hecho al ingreso del paciente a la Unidad y la última anotación realizada antes de su fallecimiento; porsupesto sin saber que esa anotación sería la última. Para la tabulación y el análisis de los datos se utilizó el paquete estadístico EPI-INFO.

\section{Resultados}

\section{Características de los pacientes atendidos y lugar de procedencia.}

En relación con la edad y sexo de los pacientes atendidos, se encontró que 46 de los pacientes atendidos eran hombres (tabla $1,56.1 \%$ ), superando por 10 el número de mujeres(36). Con respecto a la edad, la mayoría de los pacientes estan en el grupo de 61 años o más $(68.3 \%)$

Con respecto al lugar de procedencia (tabla 2), el $43.9 \%$ de los pacientes (36), procedían del cantón central de Cartago, seguidos, aunque en menor proporción, por los cantones de Tejar, Oreamuno y Paraíso, tanto hombres como mujeres.

\section{Lugar de refencia de los pacientes atendidos.}

$\mathrm{Al}$ analizar el lugar de referencia de los pacientes atendidos se encontró que el Servicio de Cirugía del Hospital Max Peralta es el sitio que más pacientes refirió: 25 (lo que representa un $30.5 \%$ ), seguido de Medicina Interna con 19 pacientes $(23.2 \%)$, el Hospital Calderón Guardia con 16 pacientes $(19.5 \%)$, de los cuáles 8 provenían de Oncología, 6 de Ginecología y 2 de la Clínica del dolor; Medicina General de las Areas de Salud refirió 15 pacientes (18.3\%), Ginecología 4 pacientes $(4.9 \%)$ y 3 pacientes fueron captados directamente por miembros de la Unidad (3.6\%).
TABLA 1

Distribución de los pacientes por edad y sexo atendidos en la Unidad de Cuidados Paliativos, Cartago, 1996

\begin{tabular}{lcccc}
\hline \multicolumn{1}{c}{ Edad } & \multicolumn{2}{c}{ Hombres } & \multicolumn{2}{c}{ Mujeres } \\
& $\mathrm{N}$ & $(\%)$ & $\mathrm{N}$ & $(\%)$ \\
\hline Más de 61 años & 31 & $(37.8)$ & 25 & $(30.5)$ \\
51 a 60 años & 11 & $(13.4)$ & 3 & $(3.7)$ \\
41 a 50 años & 3 & $(3.7)$ & 7 & $(8.5)$ \\
31 a 40 años & 1 & $(1.2)$ & 1 & $(1.2)$ \\
20 años & 0 & $(0)$ & 0 & $(0)$ \\
21 a 30 años & 0 & $(0)$ & 0 & $(0)$ \\
TOTAL & 46 & $(56.1)$ & 36 & $(43.9)$ \\
\hline
\end{tabular}

TABLA 2

Procedencia de los pacientes atendidos en la Unidad de Cuidados Paliativos. Cartago, 1996

\begin{tabular}{lcccc}
\hline \multicolumn{1}{c}{ Procedencia } & \multicolumn{2}{c}{ Hombres } & \multicolumn{2}{c}{ Mujeres } \\
& N & $(\%)$ & N & $(\%)$ \\
\hline Cartago & 21 & $(25.6)$ & 15 & $(18.3)$ \\
El Tejar & 9 & $(11.0)$ & 2 & $(2.4)$ \\
Oreamuno & 5 & $(6.1)$ & 9 & $(11.0)$ \\
Paraiso & 4 & $(4.9)$ & 4 & $(4.9)$ \\
Alvarado & 3 & $(3.7)$ & 2 & $(2.4)$ \\
Tres Rios & 2 & $(2.4)$ & 1 & $(1.2)$ \\
Otro & 2. & $(2.4)$ & 2 & $(3.7)$ \\
TOTAL & 46 & $(56.1)$ & 36 & $(43.9)$ \\
\hline
\end{tabular}

3. Diagnóstico de ingreso a la Unidad.

Del total de pacientes atendidos, $50(60.9 \%)$ ingresaron con el diagnóstico de cáncer del aparato digestivo; el cáncer gástrico fue el diagnóstico en 35 de ellos (42.6\%), seguido por cáncer de esófago, páncreas, hígado, colon y recto, en menor proporción.Con respecto a la distribución por sexo de estos diagnósticos, hubo un predominio en los hombres $(37.7 \%)$, en comparación con las mujeres (23.2\%).

El segundo lugar lo ocupa los tumores malignos del aparato reproductor femenino (cáncer de mama, cervix y ovario), con 10 casos, lo que representa el $12,1 \%$ y las enfermedades crónicas (secuelas de accidente vascular cerebral, insuficiencia renal crónica terminal, artritis reumatoide deformante) en iguales proporciones, 10 casos $(12.1 \%)$, de los cuales 7 fueron hombres y 3 mujeres. (Ver tabla 3 ).

Otros tipos de tumores (próstata, pulmón, óseo, hematológico y sistema nervioso central), guardan entre sí una proporción parecida, alrededor de 3 casos en cada diagnóstico. Solo se atendío un paciente con Síndrome de Inmunodeficiencia
Adquirida.

\section{Registro de síntomas.}

El síntoma principal de los pacientes al ingreso a la Unidad fue el dolor (70 pacientes, lo que representa el $85.4 \%$; ver tabla 4); 
TABLA 3

Relación entre diagnóstico de ingreso y sexo de los pacientes atendidos en la Unidad de Cuidados Paliativos de Cartago 1996

\begin{tabular}{|c|c|c|c|c|c|}
\hline \multirow[t]{2}{*}{ Diagnóstico } & \multicolumn{2}{|c|}{ Hombres } & \multicolumn{2}{|c|}{ Mujeres } & \multirow[t]{2}{*}{ TOTAL } \\
\hline & $\mathrm{N}$ & $(\%)$ & $\mathrm{N}$ & (\%) & \\
\hline $\begin{array}{l}\text { Tumores malignos del } \\
\text { aparato digestivo:* } \\
\text { Cáncer gástrico, esófago, }\end{array}$ & & & & & \\
\hline páncreas, hígado cólon y recto. & 31 & $(37.7)$ & 19 & (23.2) & 50 \\
\hline $\begin{array}{l}\text { Tumores malignos del aparato } \\
\text { reproductor femenino: }{ }^{* \star}\end{array}$ & & & & & \\
\hline Cáncer cérvix, ovario y mama. & 0 & (0) & 10 & $(12.1)$ & 10 \\
\hline Enfermedad crónica*** & 7 & $(8.5)$ & 3 & (3.6) & 10 \\
\hline Cáncer pulmón & 3 & (3.7) & 0 & $(0)$ & 3 \\
\hline Cáncer próstata & 3 & $(3.7)$ & 0 & (0) & 3 \\
\hline Cáncer Sistema Nervioso Central & 2 & $(2.4)$ & 1 & $(1.2)$ & 3 \\
\hline Cáncer hematológico & 0 & $(0)$ & 1 & (1.2) & 1 \\
\hline Cáncer óseo & 1 & $(1.2)$ & 0 & (0) & 1 \\
\hline SIDA & 0 & $(0)$ & 1 & $(1.2)$ & 1 \\
\hline
\end{tabular}

*Cáncer gástrico representa del total el $28 \%$ en hombres y el $15 \%$ en mujeres para un total en ambos sexos de 35 casos.

"Cérvix 4 casos, mama 5 casos y ovario 1 caso.

"... Secuelas de accidente vascular cerebral, insuficiencia renal terminal, artritis reumatoide deformante.

TABLA 4

Principales síntomas presentados por los pacientes a su ingreso en la Unidad de Cuidados Paliativos de Cartago, 1996

\begin{tabular}{lll}
\hline \multicolumn{1}{c}{ Síntomas } & N & (\%) \\
\hline Dolor & 70 & $(85.4)$ \\
Náusea y vómito & 27 & $(32.9)$ \\
Constipación & 24 & $(29.3)$ \\
Complejo sintomático de & & \\
ascitis, edemas, ictericia y prurito & 22 & $(26.8)$ \\
Síntomas & & \\
Psiquiátricos: depresión, insomnio o ansiedad & 17 & $(27.0)$ \\
Anorexia & 11 & $(13.4)$ \\
Debilidad & 10 & $(12.2)$ \\
Tos & 4 & $(4.9)$ \\
Disnea & 3 & $(3.7)$ \\
\hline
\end{tabular}

22 marzo 1998, AMC, vol. 40 (1) 
TABLA 5

Valoración del dolor de los pacientes atendidos en la Unidad de Cuidados Paliativos de Cartago, al ingreso, y la registrada antes de fallecer, Cartago, 1996

\begin{tabular}{llcccc}
\hline \multicolumn{1}{c}{ *Intensidad del Dolor } & \multicolumn{2}{c}{$\begin{array}{c}\text { Primera valoración } \\
\text { al ingreso }\end{array}$} & \multicolumn{3}{c}{$\begin{array}{c}\text { Ultima valoración } \\
\text { antes de fallecer } \\
(\%)\end{array}$} \\
\hline SIN DOLOR & $\mathrm{N}$ & $(\%)$ & $\mathrm{N}$ & $(52.4)$ \\
DOLOR LEVE & 8 & $(9.7)$ & 43 & $(31.7)$ \\
DOLOR MODERADO & 36 & $(43.9)$ & 26 & $(12.2)$ \\
DOLOR SEVERO & 24 & $(29.3)$ & 10 & $(3.7)$ \\
\hline
\end{tabular}

(*) Basada en la escala de valores numéricos

se evaluó su intensidad utilizando la escala visual númerica para establecer la efectividad del tratamiento en el alivio del dolor. Al compararlas se observa que hay diferencias sustanciales entre ellas, lográndose que 43 de los pacientes murieran sin dolor (52.4\%; Ver tabla 5). En relación con los que murieron con dolor se logró disminuir el número de pacientes que indicaron tener dolor leve, de 39 al ingreso a 26 antes de fallecer. Pero fue en el dolor moderado y severo donde las diferencias fueron más marcadas, por ejemplo en dolor moderado se pasó de un $29.3 \%$ (24 al ingreso, a solo un $12.2 \%, 10)$, antes de fallecer y en dolor severo se pasó de un $17.1 \%$ de (14) al ingreso, a solo $3.7 \%$ (3 pacientes) antes de fallecer.

Además del dolor se registraron otros síntomas, siguiendo en orden de importancia náusea y vómito en 27 pacientes (32.9\%), constipación en 24 (29.3\%), complejo síntomatico de ascitis, edemas, prurito e ictericia en 22 pacientes $(26.8 \%)$, insomnio, depresión o ansiedad en 17 (20.7\%); los demás síntomas menos frecuentes fueron anorexia, debilidad, tos y disnea.

\section{Manejo farmacológico del dolor y otros síntomas.}

Del total de pacientes incluídos en el estudio, 54 de ellos $(65.9 \%)$, no habían recibido ningún tipo de tratamiento previo a su ingreso a la Unidad, $13(15.8 \%)$ habían recibido tratamiento con opiodes y el resto había recibido algún analgésico no opiode.

En 69 pacientes el tratamiento del dolor se hizo aplicando el esquema de escalera analgésica de la OMS, y los 13 restantes ya tenían un esquema previo.

En relación con el uso de opiodes, se sumistró en primer lugar el laúdano en 32 de los pacientes $(45.7 \%)$, la morfina de acción corta en 18 pacientes $(25.7 \%)$, la morfina subcutánea en 10 pacientes ( $14.3 \%$ ), la metadona oral en 6 pacientes $(8.6 \%)$, y la codeína en 4 pacientes $(5.7 \%)$. Predominó la vía oral en la aplicación de medicamentos.

Con respecto al uso de coanalgésicos y otros medicamentos síntomaticos, la amitriptilina se utilizó en 49 pacientes, seguida de la metoclopramida y AINES en 37.
En cuanto a laxantes, se utilizó el agarolato en 24 pacientes. El haloperidol y la clorpromazina se utilizaron en 19 al igual que el demenhidranato.La dexametasona se utilizó en 14 pacientes. al igual que los anticonvulsivantes: clonazepan, carbamazepina y valproato de sodio. Otros medicamentos que se utilizaron en menor proporción fueron antihistamínicos, antibióticos y antiácidos.

\section{Lugar de manejo de los pacientes atendidos.}

El 100\% de los pacientes de la muestra fue atendido en el domicilio, sin embargo, 5 de ellos necesitaron hospitalización por alguna de las siguientes causas: infección, hemorragia, dificultad respiratoria y condiciones socio-económicas muy adversas. El $93.9 \%$ de los pacientes (77), no necesitaron hospitalización en ningún momento.

7. Otras intervenciones realizadas con los pacientes y sus familiares durante su permanencia en la Unidad.

7.I Información y enfermedad terminal.

$\mathrm{Al}$ ingreso a la Unidad el $100 \%$ de las familias de los pacientes conocían la condición de su familiar, la situación fue diferente con los pacientes, ya que el $60 \%$ de ellos (49), manifestó desconocer cual era su condición. Esta hecho cambió de manera importante, ya que antes de fallecer el $70 \%$ de los pacientes conocía su condición de enfermos en fase terminal.

7.2 Duración de la intervención.

El 34.1\% (28) de los pacientes permaneció en la Unidad por menos de un mes, el $19.5 \%$ (16) entre uno y dos meses, el $32.9 \%$ (27) entre 3 a 6 meses, y el $13.4 \%$ (11) más de 6 meses.

7.3 Clínicas de familia.

Con el $60 \%$ (49) de los pacientes atendidos se realizaron más de tres sesiones de trabajo con la familia con un $31.7 \%$ (26), de dos a tres sesiones y en un $8.5 \%$. (7) de los casos, se realizó solo una sesión.

7.4 Visitas domiciliares

Del total, 49 pacientes $(59.7 \%)$ recibieron una visita 
domiciliar por parte del equipo de la Unidad, 17 pacientes (20.7\%), de dos a tres visitas y $8(9.8 \%)$ más de tres visitas, igual proporción de pacientes no recibió ninguna visita pues su permanencia fue muy corta y no hubo tiempo para realizarla.

\section{Discusión}

\section{Características de los pacientes atendidos.}

Durante 1996 se atendió un total de 154 pacientes, número muy significativo, considerando que el equipo dedica menos de una cuarta parte de su tiempo laboral a esta actividad (solo un día a la semana); esa suma corresponde a lo que establecen los parámetros internacionales de cuidados paliativos para un equipo a tiempo completo (150 pacientes por año). ${ }^{8}$

Lo anterior implica que para cubrir este número de pacientes, gran parte de las intervenciones deben realizarse extrahorario y como trabajo voluntario.

El hecho de que la mayoría de los pacientes que fueron atendidos sean mayores de 61 años y que predomine el diagnóstico de cáncer, podría explicarse por la prevalencia nacional de este tipo de patología en ese grupo etáreo. ${ }^{9}$ Por otro lado, el no atender niños se debe a que estos son referidos a la Unidad de Cuidados Paliativos del Hospital Nacional de Niños. Considerando la tendencia a envejecer que tiene la población del país, es predecible que en el futuro aumente la demanda de este tipo de servicio lo que plantea la necesidad de fortalecer con más recursos y en ampliar en número las unidades de cuidados paliativos.

La Unidad de Cuidados Paliativos alcanzó a cubrir la demanda de atención en los pacientes en fase terminal provenientes de todos los cantones de la provincia y aunque no fue un aspecto que se registro formalmente en la investigación, es importante mencionar que se logró coordinar con las diferentes areas de salud y sus EBAIS la atención de estos pacientes.

En alguna medida el trabajo desarrollado estableció un modelo organizacional por niveles de atención, que podría servir para el desarrollo de los cuidados paliativos en otras zonas del país, a partir de esta experiencia que incorporó las estrategias planteadas por el modelo readecuado de Servicios de Salud.

\section{Lugar de referencia de los pacientes atendidos.}

$\mathrm{Al}$ analizar los lugares de procedencia de los pacientes atendidos queda claro que el trabajo desarrollado por la Unidad es una respuesta a las necesidades de los diferentes servicios clínicos y de otros hospitales, para la atención de pacientes con enfermedad terminal.

Este hecho valida la labor del equipo de cuidados paliativos, legitima la calidad de la atención y la disminución de los costos, como analizaremos más adelante.

\section{Diagnósticos de ingreso.}

El hecho de que prevalezcan los diferentes tipos de cáncer como diagnóstico de ingreso a la Unidad, coincide con la experiencia de las Unidades de Cuidados Paliativos de otros países. ${ }^{1-3.8} \mathrm{El}$ cáncer es una enfermedad que con frecuencia lleva al paciente a situaciones de incurabilidad, sobretodo en nuestro medio, en el cual su diagnóstico se realiza en estadios avanzados, y son estos pacientes los que presentan un complejo cuadro que demanda intervención, no solo en aspectos puramente biológicos, sino que sus necesidades también abarcan otras esferas como la psicológica, la social y la espiritual..$^{1.3 .8}$

Vale la pena mencionar que en algunas pacientes con cáncer del aparato reproductor que pudieron ser curadas, su diagnóstico se hizo muy tardiamente, lo que refleja fallas en cuanto a su detección temprana.

\section{Registros de Síntomas.}

Al igual que con los diagnósticos y en relación con los síntomas también, hay coincidencia con la experiencia en otros lugares donde se atienden pacientes terminales. ${ }^{1,2,4-7}$

El dolor ocupa el primer lugar, siendo a la vez prioritario su manejo, dada importancia. Basándose en los resultados obtenidos se concluye que:

4.1 El uso de la escala de valores numéricos para la evaluación del dolor y de la terapia usada demostró ser un instrumento útil y de fácil aplicación.

4.2 Protocolizar el manejo del dolor por cáncer,en este caso, siguiendo los lineamientos de la Esacalera Analgésica propuesta por la OMS (3), facilita el abordaje terapéutico y permite la obtención de resultados positivos como lo demuestra el estudio. Sin embargo esta no es una investigación del área de la terapéutica farmacológica, por lo que habría que realizar una investigación específica para determinar la efectividad de la escalera analgésica; en este trabajo es probable que el éxito logrado en el alivio del dolor no dependió unicamente por el uso de fármacos.

En relación con los otros síntomas, lo que muestran es lo complejo y aparatoso del cuadro clínico en este tipo de pacientes, ${ }^{4-7}$ siendo un verdadero reto su manejo. Lamentablemente, por limitaciones metodológicas no se registró la evolución de todos los síntomas en los pacientes atendidos, focalizándonos unicamente en el dolor. Este aspecto es un área de estudio para investigaciones futuras.

\section{Manejo farmacológico del dolor y otros síntomas.}

Los resultados demuestran, como señalamos anteriormente, la importancia de protocolizar el abordaje terapéutico del dolor y otros síntomas, sobre todo para un uso racional de los medicamentos. $^{3}$

En la mayoría de los pacientes se aplicó el esquema de escalera analgésica con buenos resultados, sin embargo, en el manejo del dolor por cáncer no existen esquemas rígidos, el éxito alcanzado en su alivio va más allá de un simple abordaje farmacológico; el dolor por cáncer demanda otro tipo de intervenciones por ser un síntoma que involucra aspectos no solo biológicos, sino psico-sociales y espirituales. ${ }^{1-3}$ El alivio depende además de medicamentos, de una relación 
cercana, empática, respetuosa y cálida con el paciente, involucrando activamente en su cuidado a sus familiares, y en algunos casos, a la comunidad.

Se podría concluir que el alivio sintomático del dolor alcanzado con los pacientes atendidos es producto de todo el proceso de intervención en su conjunto, y que involucra tanto las medidas farmacológicas como las intervenciones en las esferas psicosociales.

Mantener la vía oral como principal medio de aplicación de medicamentos, ${ }^{3-7}$ como se logró en este caso, facilita su prescripción en el nivel ambulatorio y está en relación con la calidad de vida.

Por otro lado, el manejo del dolor desde el punto de vista físico, con el uso de opiodes, no garantiza por sí solo un control adecuado de este, ${ }^{2,7}$ de ahí la importancia de utilizar los coanalgésicos y otros medicamentos coadyuvantes, tal como se hizo en el manejo de los pacientes.

Los medicamentos utilizados (con excepción del laudano, que ya no esta disponible y de la metadona que no está en el cuadro básico de la C.C.S.S), estan en el cuadro básico de la institución y son accesibles en todas las farmacias de la C.C.S.S.

\section{Lugar de manejo de los pacientes atendidos.}

El hecho de que más del $90 \%$ de los pacientes fueran cuidados y murieran en su casa es quiza uno de los aspectos más relevantes de la intervención de la Unidad, y está en relación con:

6.1 Calidad de vida, según los principios de los cuidados paliativos ${ }^{1.3}$ morir en el medio habitual rodeado y cuidado por los seres queridos es un aspecto fundamental para una muerte digna.

El fundamento de "Familia como unidad de cuidado y manejo del paciente", es todo un proceso al cual el equipo se aboca, y que implica capacitación y entrenamiento del núcleo familiar, paralelo al suministro de los insumos necesarios para mantener al paciente en casa (camas ortopédicas, colchones de agua, oxígeno, etc).

6.2 En el empleo de los servicios de salud es lógico suponer el ahorro en costos de hospitalización, en términos de díascama-hospital, y todos los demás gastos que implica hospitalizar a un paciente en estas condiciones.

\section{Otras intervenciones realizadas por la Unidad.}

La variedad de intervenciones que realizó la Unidad de Cuidados Paliativos demuestra por sí sola, las demandas de los pacientes que enfrentan una enfermedad terminal, lo que refleja lo complejo de su situación. Estas intervenciones no podrían ser realizadas por un profesional específico, lo que implica un trabajo en equipo.

\subsection{Información y enfermedad terminal.}

Según las recomedaciones de los expertos en cuidado paliativos, ${ }^{1-3}$ el hecho de que un paciente que enfrenta una enfermedad terminal conozca su condición, facilita el proceso de intervención, ligado a un desenlace menos doloroso y traumático, sin embargo, como lo demuestra este estudio, la mayoría de los pacientes que fueron atendidos desconocían su situación, lo que se modificó durante el abordaje del equipo.

En nuestro medio existe una tendencia en el personal de salud y especialmente en los médicos tratantes durante el período inicial de la enfermedad, de engañar al paciente y crear falsas expectativas, en la mayoría de las veces en común acuerdo con los familiares cercanos.

Modificar este aspecto fue un verdadero reto para el equipo e implicó un abordaje muy individualizado y cercano con el paciente y su familia, lo que al final facilitó la intervención, logrando mantener al paciente más tranquilo, en paz y en algunos casos participando activamente en su cuidado. tomando decisiones en conjunto con su familia y el equipo de cuidados paliativos.

\subsection{Duración de la intervención.}

Para la mayoría de los pacientes $(86.5 \%)$, su permanencia en la Unidad tuvo una duración de uno a seis meses, esto coincide con lo que recomiendan los expertos de cuidados paliativos y con la definición de paciente terminal, ${ }^{1-3} \mathrm{en} \mathrm{el}$ cual la expectativa de vida no debe ser mayor de seis meses. En algunos casos los pacientes fueron referidos tardiamente, lo que limitó la intervención, mientras que en otras ocasiones fueron referidos pacientes que no eran terminales. En este sentido debería procurarse sensibilizar y capacitar al personal de salud del ámbito hospitalario. en cuanto a aspectos básicos del cuidado paliativo.

\subsection{Clínicas de familia y visitas domiciliares.}

Estas dos actividades son fundamentales para lograr una adecuada intervención con los pacientes y sus familias, constituyéndose en los ejes estratégicos del equipo. Si bien es cierto se registraron unicamente estas actividades de manera cuantitativa, el equipo sabe que este tipo de intervenciones tiene gran impacto para el paciente y su familia. Además de ser un medio invaluable para determinar los recursos y limitaciones del ámbito familiar, estos aspectos se constituyen en áreas de investigaciones futuras. Las coberturas alcanzadas en estas dos actividades nos señalan también que las intervenciones en cuidados paliativos demandan gran cantidad de tiempo; en el caso de las visitas domiciliares y clínicas de familia, por ejemplo, se realizaron más de 130 visitas domiciliares y más de 280 sesiones de familia, siendo imposible de visitar a aquellos pacientes que fueron referidos tardíamente.

\section{Abstract}

Palliative care is a relative new activity in Costa Rica. The Palliative Care Unit of Max Peralta Hospital in Cartago is the first team of the country that began the attention of terminal patients and their families in 1990. This is a homecare program and the team is integrated by doctors, nurses, pharmachologists, psychologists and volunteers. This article 
describes the activities the Palliative Care Unit of Max Peralta Hospital did during 1996.

\section{Agradecimiento}

$\mathrm{Al}$ equipo profesional y al grupo de voluntarios de la Unidad de Cuidados Paliativos de Cartago, gracias a su esfuerzo es posible llevar un poco de alivio y aliento a los pacientes en fase terminal y sus familiares, labor que trata de describir este artículo. Al Dr Carlos Cerdas Mena, médico interno, por haber consolidado muchos de los datos. A la Lic Xinia Fernández Vargas y a la Dra Virginia Cozzy por sus valiosas sugerencias en la redacción del texto.
Referencias
1. Saunders C, Bines M, Living with Dying, The managment of terminal 1- Saunders C, Bines
disease,England, 1983.

2- Bejarano P, Jaramillo de Isa, Morir con dignidad, Fundamentos del Cuidado Paliativo.Colombia: Amazones Editores Ltda,1992.

3- Comité de expertos de la OMS, Alivio del dolor y tratamiento paliativo por cáncer, Serie de Informes Técnicos 804, OMS.Ginebra,Suiza, 1990.

4- American Pain Society,Principles of Analgesic Use in Treatment of Acute Pain and Cancer Pain.USA third Edition,1992.

5ain and Cancer Pain.USA thira Edition, 1992 . 5- Bruera E, Manejo farmacológic
Cuidados Paliativos.México,1992.

6. C. Stratton Hill Jr, Pain control in the patient with cancer, Cancer J for Clinicians, 1988; Vol 38:6-12.

7. Foley K.Payme R.Terapéutica del dolor, México: Interamericana Mc

Graw-Hill,1992. 8standards de Cuidados Paliativos, Cuadernos de Cuidados Estandards de Cuidados

Paliativos.Cataluña,España, 1992 . Descriptiva,Editorial de la Universidad de Costa Rica, San José, Costa Rica,1995.

26 marzo 1998, AMC, vol. 40 (1) 